\title{
Reducing Polarization Dependent Loss of Silicon-on-Insulator Fiber to Chip Grating Couplers
}

\author{
Robert Halir, Diedrik Vermeulen, and Günther Roelkens
}

\begin{abstract}
For many telecommunication applications, low Polarization Dependent Loss (PDL) operation is mandatory. On the silicon-on-insulator photonic wire platform this can be achieved using polarization diversity configurations. However, as we show here, when using two dimensional grating couplers for near vertical fiber input and output, the low PDL bandwidth is limited. We propose and demonstrate the use of a $\pi$ phase shifter in one of the arms of the polarization diversity circuit to effectively reduce PDL (0.15 dB PDL is shown) and increase the low PDL bandwidth.
\end{abstract}

\section{INTRODUCTION}

$\mathbf{S}^{\mathrm{n}}$ ILICON-on-insulator photonic wire technology allows for a substantial scaling in the size of photonic integrated circuits due to the high refractive index contrast that is available. Silicon waveguides with dimensions on the order of $500 \mathrm{~nm}$ by $200 \mathrm{~nm}$ allow for single mode propagation and tight optical confinement. The high refractive index contrast makes the waveguide structures very polarization dependent however, which is an issue in many practical applications. Therefore polarization diversity schemes are being proposed to tackle this problem. In one approach, a polarization splitter and rotator is being integrated on the chip, such that the two arms of the polarization diversity circuit are processing the same polarization state. This resulted in relatively low PDL (about $1 \mathrm{~dB}$ ) [1], [2]. In [3], it was proposed to use a two-dimensional grating structure, which at the same time fulfills the role of optical fiber interface, polarization splitter and rotator, all on a $100 \mu \mathrm{m}^{2}$ area. Taking into account the full bandwidth of the grating coupler spectrum, still quite substantial polarization dependent loss can be observed in the experiment ( 1 to $2 \mathrm{~dB}$ PDL in the fiber to fiber coupling $1 \mathrm{~dB}$ bandwidth). In this paper we show that, by introducing a $\pi$ phase shifter in one arm of the polarization diversity circuit, a substantial polarization dependent loss (PDL) reduction can be obtained.

\section{CAuses And Reduction Of PDL}

In a polarization diversity scheme like the one shown in Fig. 1, light is coupled in and out of the circuit with fibers

This work was carried out in the framework of the Dutch SmartmixMemphis project. Robert Halir acknowledges the Spanish Ministerio de Educación for the FPU scholarship with reference AP-2006-03355. D. Vermeulen thanks the Institute for the Promotion of Innovation by Science and Technology in Flanders (IWT) for a grant. G. Roelkens acknowledges the Fund for Scientific Research Vlaanderen (FWO) for a postdoctoral grant.

Robert Halir is with the Departamento de Ingeniería de Comunicaciones, ETSI Telecomunicación, Universidad de Málaga, 29010 Málaga, Spain (email: robert.halir@ic.uma.es).

Diedrik Vermeulen and Günther Roelkens are with the Department of Information Technology (INTEC), Ghent University - Interuniversity Microelectronics Center (IMEC), B-9000Gent, Belgium (e-mail: gunther.roelkens@intec.ugent.be)(website: http://photonics.intec.ugent.be)

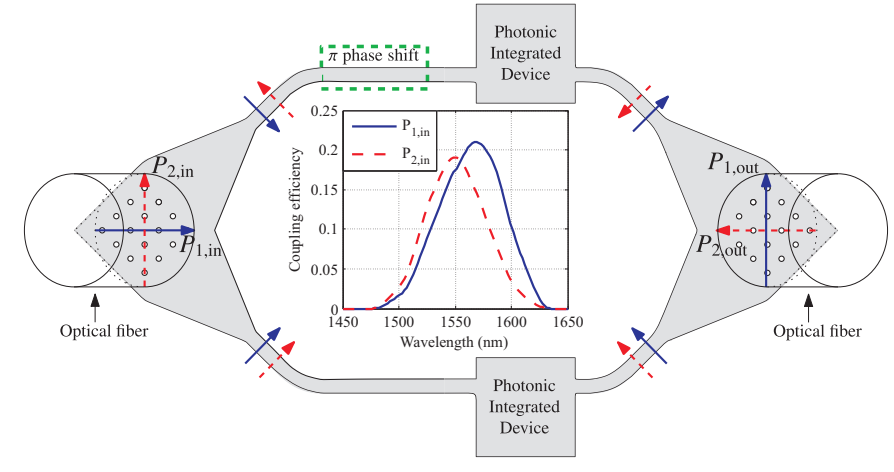

Fig. 1. Schematic illustration of a polarization diversity circuit with 2D gratings. The inset shows the simulated coupling efficiency of a $2 \mathrm{D}$ grating for polarizations $P_{1, \text { in }}$ and $P_{2, \text { in }}$.

positioned over the two dimensional grating couplers [3], [4]. These couplers convert any polarization state in the fiber to TE polarized light in both arms of the polarization diversity circuit. In order to avoid strong back reflections from the gratings, the fibers have to be slightly tilted, typically at $\sim 10^{\circ}$ with respect to the vertical. In both arms the light is then directed to the integrated photonic device that performs the desired functionality and is finally coupled into the output fiber using an identical 2D grating coupler. In such a polarization diversity configuration there are three main sources of PDL.

First, if the input and output fibers are not positioned along the diagonal of the 2D grating couplers, symmetry is lost and some polarization states will couple more efficiently to and from the integrated waveguides than others. This contribution to PDL can be minimized with proper fiber alignment, as detailed in section III. Second, due to fluctuations during fabrication the devices in either arm may not behave identically, thus introducing a different amount of loss. Trimming or tuning of the devices may be used to cancel this effect. In some device configurations the same device can be used for both arms of the polarization diversity circuit, by propagation in opposite directions [5]. Finally, the grating couplers themselves also introduce PDL. This is best understood considering the two linear polarizations at $0^{\circ}$ and $90^{\circ}, P_{1, \text { in }}$ and $P_{2, \text { in }}$, shown in Fig. 1. Due to the near vertical coupling, $P_{1 \text {,in }}$ is slightly tilted out of the grating plane, while $P_{2, \text { in }}$ lies in the same plane as the grating coupler. Consequently $P_{1, \text { in }}$ and $P_{2, \text { in }}$ will not exhibit the same coupling efficiency. The different coupling efficiencies are shown in the inset of Fig. 1. They were obtained with 3D Finite Difference Time Domain simulations of a $19 \times 19$ array of circular holes with a diameter of $400 \mathrm{~nm}$ and a $605 \mathrm{~nm}$ pitch. The outgoing waveguides form 
an $83.8^{\circ}$ angle to account for the effect of the tilted fiber (see [4]). Since the coupling efficiency of both polarizations only coincides at a specific wavelength, the grating coupler will generally introduce PDL. The reduction of this type of PDL is the main contribution of this paper and will be discussed in more detail in the following.

From symmetry considerations it is clear that if polarization state $P_{1, \text { in }}$ is aligned along the grating bisection line, it will couple symmetrically to the upper and lower arm of the polarization diversity circuit. This is schematically illustrated with the solid arrows on the left side of Fig. 1. On the other hand, polarization state $P_{2 \text {,in }}$ will couple antisymmetrically, i.e. with a $180^{\circ}$ phase shift between the upper and the lower arm, as shown with the dashed arrows on the left side of Fig. 1. If the optical signals in both arms experience the same phase shift when propagating through the photonic device, the output grating will restore the original input polarization state. That means that when light with either polarization $P_{1, \text { in }}$ or $P_{2, \text { in }}$ is coupled in, it will experience the same coupling efficiency curve (see inset of Fig. 1) twice, thus increasing PDL.

Here we propose to introduce a $\pi$ phase shift in one of the arms of the polarization diversity circuit. As illustrated in on the right side of Fig. 1, this will cause the polarization states $P_{1, \text { in }}$ and $P_{2, \text { in }}$ to interchange their orientation at the output grating coupler. Consequently, either of these states will experience both coupling efficiency curves (one at the input, and the other one at the output), so that the overall input to output coupling is identical for both, and PDL vanishes. Naturally, complete PDL compensation requires almost identical input and output coupling efficiencies. This can be achieved because the grating couplers are closely together and are defined by state-of-the-art $193 \mathrm{~nm}$ DUV lithography, so that they are virtually identical, and highly symmetric fiber alignment is possible using one dimensional grating couplers as polarization references (see section III).

\section{Device and Measurement Setup}

To demonstrate the PDL reduction concept we use a polarization diversity circuit as shown in Fig. 1, with the generic device substituted by a ring resonator (see inset of Fig. 2). The final purpose of the circuit is to act as tunable wavelength router for FTTH applications. As such, in this prototype it has to deliver two wavelengths to the home user, one with the downstream data $\left(\lambda_{\text {down, } 1,2}\right)$, and a continuous wave $\left(\lambda_{\mathrm{cw}, 1,2}\right)$ that can be modulated at the user end. Both of these wavelengths must be routed with low PDL. Furthermore, wavelengths intended for other users should be suppressed, but PDL is less critical at these wavelengths. The component operates with the downstream data and continuous wave wavelengths at two consecutive transmission peaks of the ring resonators, while wavelengths of other users are located at transmission minima. The recorded transmission spectrum is plotted in 2. An insertion loss of $21 \mathrm{~dB}$ is obtained, which is due to the limited efficiency of the grating couplers (approximately $7 \mathrm{~dB}$ loss per coupler) and the $1 \times 4$ splitter implemented in the circuit. The efficiency of the grating couplers can be improved however by using a silicon overlay, as shown in [6].

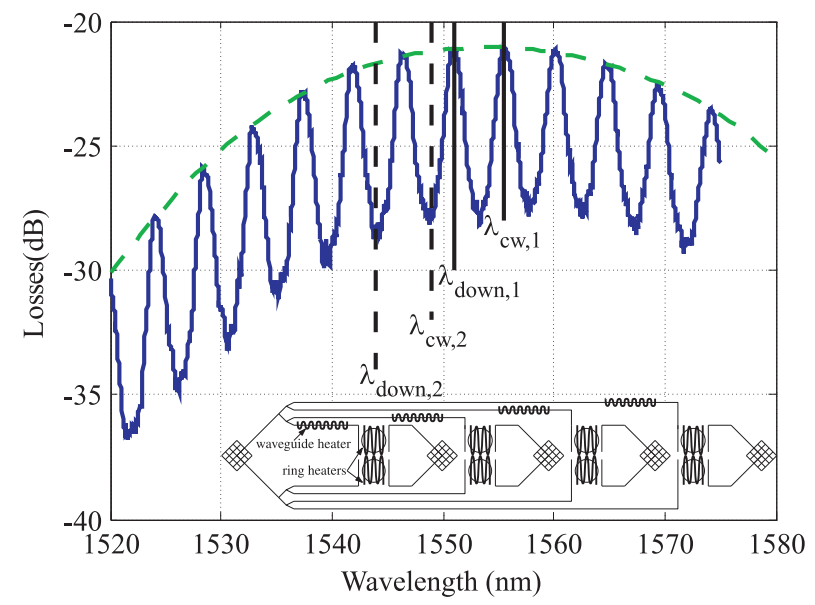

Fig. 2. Measured spectral response of the wavelength router with all heaters turned off. The dashed envelope indicates the overall efficiency of the two 2D grating couplers. A schematic of the device is shown in the inset.

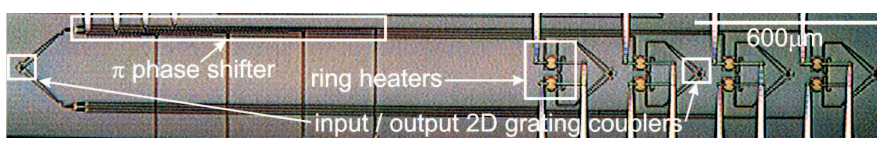

Fig. 3. Microscope image of the wavelength router with waveguide heaters (phase shifters) and ring heaters.

This, however, does not change the concept of PDL reduction. The free spectral range of the rings is $\sim 4.5 \mathrm{~nm}$, while their extinction ratio will still be optimized.

The device was fabricated on a SOI wafer with a silicon thickness of $220 \mathrm{~nm}$ using deep UV lithography and a two etch depth process. A $70 \mathrm{~nm}$ etch depth was used for the grating couplers, whereas the photonic wires were completely etched. After cladding the device with DVS-BCB, titanium heaters were defined on top of both ring resonators to allow for wavelength tuning as well as matching the devices in both arms. The $\pi$ phase shifter was also implemented with a heater on top of one of the arms of the polarization diversity circuit. A microscope image of a wavelength router capable of addressing four users is shown in Fig. 3.

To reduce fiber-misalignment induced PDL, the fibers were first aligned to a reference waveguide with one dimensional grating couplers. The high polarization dependence of these couplers was used to adjust the input polarization to the $P_{2, \text { in }}$ state shown in Fig. 1, using a polarization controller. The fibers were then aligned for maximum transmission on the 2D gratings. Since the fiber coupling efficiency is relatively position tolerant (typically $\sim 2 \mu \mathrm{m}$ for a $1 \mathrm{~dB}$ variation), this effectively minimizes PDL due to asymmetric power coupling. However, a slight misalignment, that does not produce significant coupling asymmetry, may still result in an unwanted phase shift between both arms. Current was supplied independently to the $\pi$ phase shifter and the ring heaters. For PDL characterization a tunable laser source and the Fiberpro "PL 2000" PDL meter ${ }^{1}$ were employed. The latter employs the polarization scanning method to determine PDL and allows for simultaneous insertion loss measurements.

\footnotetext{
${ }^{1}$ Available: http://www.fiberpro.com/products/ofc_pl2000.asp
} 


\section{Results}

For device characterization, the transmission spectrum of the device was first recorded with the fixed $P_{2, \text { in }}$ polarization state (see Fig. 2). Without changing the fiber alignment, PDL was then measured as a function of heater power at the transmission peaks using the PDL meter.

Initially, the ring heaters were switched off, and the power supplied to the waveguide heater was varied. At a wavelength of $\lambda=1556 \mathrm{~nm}$ this yields the dashed curve in Fig. 4(a). As expected, PDL decreases as the phase shift increases, reaching a minimum value of $0.5 \mathrm{~dB}$ at $14 \mathrm{~mW}$ of heating power. As more power is applied, the phase shift exceeds the optimum value so that PDL increases again. The fact that the device exhibits a higher PDL with $30 \mathrm{~mW}$ of power than without heating, can be attributed to imperfect fabrication or slight fiber misalignment that yield an initial non-zero phase shift between the arms. Only marginal variations in insertion loss of $\sim 0.2 \mathrm{~dB}$ were observed while heating the device.

The ring heaters are used to compensate for both the initial fabrication differences between the rings and differential thermal cross-talk from the waveguide arm heater. A trial and error approach was used to determine which of the two ring heaters cancels the combination of these two effects, and thus further reduces PDL. When the waveguide heater was switched off, and power was supplied only to the previously chosen ring heater (with the other ring heater off), PDL improvements were negligible. This is shown by the dashed curve in Fig. 4(b) and indicates that the initial fabrication differences between the rings were low. However, when the waveguide heater is fixed at $17.8 \mathrm{~mW}$, different thermal crosstalk is induced in the rings, so that they must be matched again with the ring heaters. This can be seen in the solid curve in Fig. 4(b), where the waveguide heater is fixed at $17.8 \mathrm{~mW}$ and PDL reaches a minimum of $0.15 \mathrm{~dB}$ at $6 \mathrm{~mW}$ ring heater power. Conversely, as shown in the solid curve in 4(a), at this power level for the ring heater the minimum PDL is achieved at $17.8 \mathrm{~mW}$ waveguide heating power. The fact that the waveguide heating powers that yield minimum PDL are slightly different depending on the power supplied to the ring heaters, can again be explained by thermal crosstalk from the ring heater to the polarization diversity waveguide arm. The $0.15 \mathrm{~dB}$ of remaining PDL are attributed to minor asymmetries in the fiber alignment.

The optimum waveguide and ring heater power levels were found to be generally slightly different for different transmission peaks. However, choosing adequate levels, it is possible to achieve low PDL at several consecutive peaks, inside the $1 \mathrm{~dB}$ fiber to fiber coupling bandwidth. This is shown in table I, from where it is clear that PDL well below $0.5 \mathrm{~dB}$ for the downstream wavelength as well as continuous wave located at the next peak is readily achieved at $\lambda=1551.5 \mathrm{~nm}$ and $\lambda=1556 \mathrm{~nm}$. Also notice PDL improvements of up to $1.4 \mathrm{~dB}$ (at $\lambda=1560.5 \mathrm{~nm}$ ) are achieved when the heaters are used. By readjusting the heaters, low PDL can be obtained in a different wavelength range.

\section{CONCLusions}

We have shown experimentally that by using a $\pi$ phase shift in one of the arms of a polarization diversity circuit
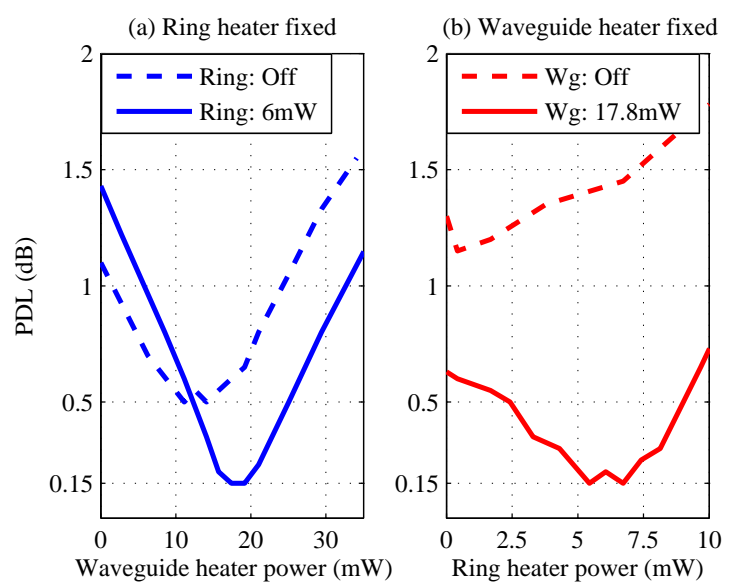

Fig. 4. Measured PDL at $\lambda=1556 \mathrm{~nm}$ as a function of waveguide and ring heater powers.

TABLE I

MEASURED PDL AT TRANSMISSION PEAKS WITH HEATERS ON AND OFF. WAVEGUIDE HEATER POWER $\sim 20 \mathrm{~mW}$, RING HEATER POWER $\sim 4 \mathrm{~mW}$.

\begin{tabular}{ccccccc}
\hline $\begin{array}{c}\text { Wavelength } \\
(\mathrm{nm})\end{array}$ & 1542.5 & 1547.0 & 1551.5 & 1556.0 & 1560.5 & 1565.0 \\
\hline $\begin{array}{c}\text { PDL }(\mathrm{dB}) \\
\text { heaters on }\end{array}$ & 0.8 & 0.4 & 0.4 & 0.35 & 0.6 & 1 \\
\hline $\begin{array}{c}\text { PDL }(\mathrm{dB}) \\
\text { heaters off }\end{array}$ & 1.25 & 1.1 & 1.2 & 1.4 & 2.0 & 2.2 \\
\hline
\end{tabular}

the PDL induced by the near vertical grating couplers can be reduced from more than $1 \mathrm{~dB}$ to only $0.15 \mathrm{~dB}$, at a fixed wavelength. This requires careful alignment of the input and output fibers and exact matching of the devices in each arm. In the wavelength router used to demonstrate this concept, it was also possible to achieve PDL below $0.5 \mathrm{~dB}$ at several consecutive transmission peaks. This technique should enable low PDL operation in a variety of polarization diversity configurations.

\section{REFERENCES}

[1] T. Barwicz, M. Watts, M. Popovic, P. Rakich, L. Socci, F. Kartner, E. Ippen, and H. Smith, "Polarization-transparent microphotonic devices in the strong confinement limit," Nat. Photonics, vol. 1, no. 1, pp. 57-60, 2007.

[2] H. Fukuda, K. Yamada, T. Tsuchizawa, T. Watanabe, H. Shinojima, and S. Itabashi, "Silicon photonic circuit with polarization diversity," Opt. Express, vol. 16, pp. 4872-4880, 2008.

[3] D. Taillaert, H. Chong, P. Borel, L. Frandsen, R. De La Rue, and R. Baets, "A compact two-dimensional grating coupler used as a polarization splitter," IEEE Photonics Technol. Lett., vol. 15, no. 9, pp. 1249-1251, 2003.

[4] F. Van Laere, T. Stomeo, D. Taillaert, G. Roelkens, D. Van Thourhout, T. Krauss, and R. Baets, "Efficient Polarization Diversity Grating Couplers in Bonded InP-Membrane," IEEE Photonics Technol. Lett., vol. 20, no. 4, 2008.

[5] W. Bogaerts, D. Taillaert, P. Dumon, D. Van Thourhout, R. Baets, and E. Pluk, "A polarization-diversity wavelength duplexer circuit in siliconon-insulator photonic wires," Opt. Express, vol. 15, no. 4, pp. 1567-1578, 2007.

[6] G. Roelkens, D. Vermeulen, D. Van Thourhout, R. Baets, S. Brision, P. Lyan, P. Gautier, and J. Fédéli, "High efficiency diffractive grating couplers for interfacing a single mode optical fiber with a nanophotonic silicon-on-insulator waveguide circuit," Appl. Phys. Lett, vol. 92, pp. 131 101-131 103, 2008. 